\title{
EVALUACIÓN DE LA BIODEGRADACIÓN DEL COLORANTE AZUL BRILLANTE UTILIZANDO HONGOS DE LA PODREDUMBRE BLANCA Y SUS CONSORCIOS
}

\section{EVALUATION OF BRILLIANT BLUE DYE BIODEGRADATION BY WHITE ROT FUNGI AND THEIR CONSORTIA}

\author{
Julián Rojas ${ }^{1}$, Angelina Hormaza ${ }^{2}$
}

\begin{abstract}
${ }^{1}$ Microbiólogo Industrial, Microbiólogo Agrícola y Veterinario, M.Sc. en Ciencias Biotecnología, Investigador del Grupo de Investigación SIRYTCOR. Universidad Nacional de Colombia, Sede Medellín, calle 59 A N 63-20, e-mail: juarojasba@unal.edu. co; ${ }^{2}$ M.Sc. en Química Orgánica, Doctora en Ciencias Químicas, Directora del Grupo de Investigación SIRYTCOR, Profesor Asociado Escuela de Química, Facultad de Ciencias. Universidad Nacional de Colombia, Sede Medellín, calle 59 A N 63-20, e-mail: ahormaza@unal.edu.co
\end{abstract}

Rev. U.D.C.A. Act. \& Div. Cient. 19(1): 179-187, Enero, Junio 2015.

\section{RESUMEN}

Los efluentes coloreados procedentes de la industria textil causan efectos adversos en los ecosistemas acuáticos, que conducen a un deterioro de su estética y a una disminución de los procesos fotosintéticos. En este trabajo, se evaluó la biodegradación del colorante textil azul brillante $A B$, sobre residuos de flores, empleando tres cepas fúngicas: Pleurotus ostreatus $(\mathrm{Po})$, Pleurotus pulmonarius $(\mathrm{Pp})$ y Trametes versicolor $(T v)$, así como algunos de sus consorcios. Las mejores condiciones, se determinaron mediante fermentación en estado sólido, con las especies fúngicas individuales y con dos de sus consorcios fúngicos: C1: Po-Tv y C2: Po-Pp, a través de un diseño factorial. Asimismo, se evaluó la cinética del proceso y se cuantificó la actividad enzimática. Se alcanzó un porcentaje de biodegradación del AB del 99,14\%, con $P p$, como cepa individual, bajo una relación de carbono:nitrógeno (C:N) de 20:1, con cuatro discos de biomasa y del 99,19\%, para el C2, bajo una relación de C:N de 40:1, con cuatro discos de biomasa, en relación 1:1 de cada hongo seleccionado. En cuanto a la cinética, se encontró que el C2 requirió 10 días para alcanzar una degradación del $92 \%$ del $A B$; este mismo porcentaje fue obtenido con $P p$ a los 12 días. Con respecto a las enzimas involucradas, se encontró que la mayor actividad fue registrada para la lacasa, tanto en cepas individuales como en los consorcios fúngicos, con valores de 6,98 ULac/gss y 17,83 ULac/gss, respectivamente.

Palabras clave: Biorremediación, colorantes trifenilmetánicos, microorganismos fúngicos, actividad enzimática.

\section{SUMMARY}

Colored effluent from the textile industry cause adverse effects on aquatic ecosystems, leading to a deterioration of their aesthetic and a decrease in photosynthetic processes. In this study, the biodegradation of textile dye brilliant blue, BB, over flowers waste using three fungal strains Pleurotus ostreatus (Po), Pleurotus pulmonarius (Pp), Trametes versicolor $(T v)$ and some of their consortia was evaluated. The best conditions of degradation were determined by solid state fermentation with individual fungal species and two of its consortia C1: Po-Tu y C2: Po-Pp, through a factorial design. Likewise, the kinetics of the process was evaluated and the enzyme activity was quantified. A biodegradation percentage from BB of $99.14 \%$ was reached by $P p$ as single strain, under a ratio of C:N of 20:1, with four biomass plugs and of $99.19 \%$ for $\mathrm{C} 2$, under a ratio of $\mathrm{C}: \mathrm{N}$ of $40: 1$, with four biomass plugs in 1:1 ratio of each selected fungus. As to the kinetics, it was found that C2 required a time of 10 days to reach $92 \%$ of $B B$ decolourisation; this same percentage was obtained by $P p$ at 12 days. Regarding to the involved enzymes, it was found that the highest activity was recorded for the laccase by both individual strains and fungal consortia, with values of 6.98 ULAC/gss and 17.83 ULAC/gss respectively.

Key words: Bioremediation, triphenylmethane dyes, fungal microorganisms, enzymatic activity.

\section{INTRODUCCIÓN}

Los colorantes sintéticos son compuestos de gran interés, por su amplio uso en la industria textil (Murugesan et al. 2007). Su implementación en los procesos de tinción gene- 
ra aguas residuales coloreadas, las cuales, son estables a la luz, a la temperatura y a ataques microbianos, confiriéndoles un carácter recalcitrante (Verma \& Madamwar, 2002; Tychanowicz et al. 2004; Fernández et al. 2009). La liberación de estos colorantes en el ecosistema acuático, conlleva a un impacto negativo, reflejado, principalmente, en el deterioro visual y en el desequilibrio dinámico del sistema, como consecuencia de la menor disponibilidad de oxígeno, debida a la notable reducción del proceso fotosintético (Chander \& Arora, 2007; Prigione et al. 2008; Saratale et al. 2009).

El azul brillante $\mathrm{AB}$ es un colorante trifenilmetánico, ampliamente utilizado en la tinción de productos textiles y de cuero, así como en la industria alimentaria en bebidas, en productos lácteos, en polvos, en gelatinas, en dulces, en helados, en jarabes, en extractos y en condimentos (Gupta et al. 2006). Por esta razón, su presencia es frecuente en los efluentes coloreados, vertidos por diferentes industrias, ocasionando impactos negativos.

En la actualidad, se cuentan con distintos métodos fisicoquímicos para el tratamiento de aguas coloreadas, que ofrecen resultados satisfactorios en la remoción de colorantes, pero su implementación genera elevados costos y lodos (Tychanowicz et al. 2004). Por lo anterior, se ha incrementado el interés en la búsqueda de métodos de tratamientos económicos y eficientes, tales como los biológicos, utilizando, en especial, hongos de la podredumbre blanca, HPB. Éstos, se caracterizan por poseer un complejo enzimático, que les permite degradar un extenso número de compuestos orgánicos recalcitrantes (Boer et al. 2004; Tychanowicz et al. 2004; Fernández et al. 2009). Los HPB poseen un sistema enzimático extracelular, que les permite realizar, naturalmente, la biodegradación de la lignina. Las enzimas que componen dicho sistema son de tipo oxidasas; dentro de ellas, las más destacadas son las lacasas, la ligninoperoxidasa (LiP), la manganeso peroxidasa $(\mathrm{MnP})$; estás dos últimas dependientes de peróxido de hidrógeno (Robinson et al. 2001; Fernández et al. 2009). Las enzimas producidas por estos hongos han sido obtenidas, tanto por fermentación en sumergido como por fermentación en estado sólido, FES; no obstante, la fermentación en sumergido no refleja las características naturales del hábitat de crecimiento de los HPB, mientras que la FES, recrea ese ambiente natural, dado que ellos crecen sobre materiales lignocelulosicos húmedos y en ausencia de agua libre (Gutiérrez et al. 1999; Tychanowicz et al. 2004). Esta última metodología ha ganado especial atención para el tratamiento de colorantes, por los considerables porcentajes de decoloración, así como por la producción de enzimas, tal como ha sido reportado por Robinson et al. (2001); Murugesan et al. (2007); Robinson \& Nigam, (2008). Las fermentaciones en estado sólido, se pueden llevar a cabo por un organismo individual o por su consorcio, es decir, utilizando dos o más organismos en el proceso (Gutiérrez et al. 1999; Malaviya \& Rathore, 2007). El establecimiento de los consorcios fúngicos está supeditado al sinergismo de las especies participantes y a las condiciones nutricionales (Rojas \& Hormaza, 2014). Al presentarse una compatibilidad positiva entre los organismos participantes en la fermentación, se puede aprovechar de forma satisfactoria el sustrato, superando las limitaciones nutricionales del soporte sólido y, a la vez, alcanzar una mayor producción enzimática (Gutiérrez et al. 1999; Kaushik \& Malik, 2009; Kausar et al. 2010; Yang et al. 2011).

Por otro lado, en Colombia se producen, aproximadamente, 1.700 toneladas de residuos de flores al año, de acuerdo con Jaramillo et al. (2013), que son tratadas, usualmente, por tecnologías, como el compostaje, generando abonos orgánicos, que pueden contener microorganismos fitopatógenos, enterobacterias y virus (Gutiérrez et al. 2009). Como alternativa a la disposición de estos residuos de flores se han realizado estudios, los cuales, han mostrado un desempeño satisfactorio de estos residuos, como potenciales adsorbentes, en la remoción del colorante AB (Jaramillo et al. 2013; Echavarria \& Hormaza, 2014). Por ello y debido a su amplia disponibilidad, el sistema residuo de flores- $\mathrm{AB}$ ha sido seleccionado como soporte sólido para la FES, del presente estudio.

Con el propósito de contribuir a la investigación relacionada con los procesos fermentativos en estado sólido y con la metodología biológica poco explorada en el país para el tratamiento de aguas coloreadas, en el presente trabajo, se evaluó la biodegradación del colorante textil AB sobre residuos de flores, empleando tres cepas fúngicas de HPB y dos de sus consorcios. Además, se determinó la cinética del proceso y se cuantificó la actividad enzimática, tanto para las especies individuales como para los consorcios, información que será utilizada para el posible escalado de este proceso de biorremediación de colorantes sintéticos.

\section{MATERIALES Y MÉTODOS}

Cepas: Fueron utilizadas tres cepas de los HPB, Pleurotus ostreatus, Pleutorus pulmonarius y Trametes versicolor, obtenidas del Laboratorio de Tejidos Vegetales de la Universidad de Antioquia, que fueron reactivadas, incubándose a $28^{\circ} \mathrm{C}$, durante diez días, en agar salvado de trigo, cuya composición fue $350 \mathrm{~g}$, salvado de trigo/L; $5,0 \mathrm{~g}$, peptona /L; $10 \mathrm{~g}$, de glucosa/L; 2,0g, de extracto de levadura/L; 0,1g, $\mathrm{KH}_{2} \mathrm{PO}_{4} / \mathrm{L} ; 0,05 \mathrm{~g}, \mathrm{MgSO}_{4} .7 \mathrm{H}_{2} \mathrm{O} / \mathrm{L}$ y $0,076 \mathrm{~g}, \mathrm{MnSO}_{4} . \mathrm{H}_{2} \mathrm{O} / \mathrm{L}$. De este medio, se tomaron discos de $10 \mathrm{~mm}$ de diámetro del borde de crecimiento del hongo, que fueron utilizados como inóculo para las pruebas de decoloración inicial y el posterior proceso de FES.

Pruebas de decoloración inicial del AB: Se utilizaron los medios agar PDA OXOID $\mathrm{pH}=5,5$ y el medio reportado por 
Radha et al. (2005), cuya composición es 2,0g, de glucosa/L; $0,075 \mathrm{~g}, \mathrm{NH}_{4} \mathrm{Cl} / \mathrm{L} ; 2 \mathrm{~g}, \mathrm{KH}_{2} \mathrm{PO}_{4} / \mathrm{L} ; 0,5 \mathrm{~g}, \mathrm{MgSO}_{4} .7 \mathrm{H}_{2} \mathrm{O} / \mathrm{L} ; 0,1 \mathrm{~g}$, $\mathrm{CaCl}_{2} .2 \mathrm{H}_{2} \mathrm{O} / \mathrm{L} ; 100 \mu \mathrm{g}$, de tiamina/L; 0,5g, $\mathrm{MnSO}_{4} . \mathrm{H}_{2} \mathrm{O} / \mathrm{L}$; $0,1 \mathrm{~g}, \mathrm{FeSO}_{4} .7 \mathrm{H}_{2} \mathrm{O} / \mathrm{L} ; 0,1 \mathrm{~g}, \mathrm{ZnSO}_{4} .7 \mathrm{H}_{2} \mathrm{O} / \mathrm{L} ; 18 \mathrm{~g}$, de agaragar/L y se ajustó el pH a 4,5, donde se disolvió el $\mathrm{AB}$ a una concentración de 100ppm. Cada uno de estos medios fue servido respectivamente en tres cajas de Petri de $9,0 \mathrm{~cm}$ de diámetro, a las cuales, se adicionó un disco de $0,5 \mathrm{~cm}$ de diámetro, de cada una de las cepas de estudio. Seguidamente, las cajas fueron sometidas a incubación por diez días, a $28^{\circ} \mathrm{C}$.

La selección del mejor medio, se apoyó en la apreciación cualitativa de la decoloración y en el mayor crecimiento en centímetros de las tres especies fúngicas evaluadas. Adicionalmente, se llevó a cabo la prueba del cosustrato de carbono, con el fin de determinar la influencia de una fuente de carbono en la degradación de AB. Para ello, se empleó $\mathrm{AB}$ a 100ppm, el medio seleccionado en la prueba anterior, sin glucosa y suplementado con glucosa, utilizando los tres hongos individuales, observando el proceso de decoloración durante diez días. El criterio de selección fue la apreciación visual de decoloración mostrada por las especies fúngicas de estudio.

Preparación del soporte sólido: Los residuos de flores, RF, utilizados corresponden a los tallos de margarita, de rosa y de clavel, en relación 1:1:1. Su tratamiento incluyó lavado con agua destilada, secado a $80^{\circ} \mathrm{C}$, durante 48 horas, molienda y tamizado, bajo las condiciones previamente descritas por Jaramillo et al. (2013). El colorante AB fue removido de una solución coloreada, simulada bajo las condiciones óptimas del proceso de adsorción en sistema discontinuo, descritas en Jaramillo et al. (2013).

FES con hongos individuales: Los ensayos fueron llevados a cabo en Erlenmeyer de $50 \mathrm{~mL}$, utilizando un diseño estadístico factorial de niveles mixtos $3 \times 2 \times 2$, con tres réplicas por tratamiento. Los factores considerados fueron hongo, definido por las tres especies evaluadas: P. pulmonarius, $P$. ostreatus y $T$. versicolor, el factor biomasa, dado por los niveles de 2 y 4 discos de agar, colonizados por los hongos y el factor relación C:N, dado por los niveles 40:1 y 20:1. Se seleccionaron 4 discos de inóculo, con el fin de garantizar la colonización total de la superficie del recipiente, donde se lleva a cabo la FES. La utilización de dos discos de inóculo posibilita evaluar la influencia de la cantidad de biomasa en el proceso. Con respecto a la relación $\mathrm{C}: \mathrm{N}$, se ha reportado que influye en la producción enzimática de los HPB. Tychanowycz et al. (2004) encontraron una máxima producción de lacasa, con la relación 30:1, por ello se decidió utilizar las relaciones 40:1 y 20:1, valores cercanos a esta condición.

Se utilizaron 200mg de RF, a los cuales, se adicionó una solución mineral del medio Rhada, que contenía glucosa, clo- ruro de amonio, $2 \mathrm{~g}$ de $\mathrm{KH}_{2} \mathrm{PO}_{4} / \mathrm{L}$, 0,5g de $\mathrm{MgSO}_{4} .7 \mathrm{H}_{2} \mathrm{O} / \mathrm{L}$, $0,1 \mathrm{~g}$ de $\mathrm{CaCl}_{2} .2 \mathrm{H}_{2} \mathrm{O} / \mathrm{L}, 100 \mu \mathrm{g}$ de tiamina/L, 0,5g de $\mathrm{MnSO}_{4}$. $\mathrm{H}_{2} \mathrm{O} / \mathrm{L}, 0,1 \mathrm{~g}$ de $\mathrm{FeSO}_{4} .7 \mathrm{H}_{2} \mathrm{O} / \mathrm{L}, 0,1 \mathrm{~g}$ de $\mathrm{ZnSO}_{4} .7 \mathrm{H}_{2} \mathrm{O} / \mathrm{L}$, modificándose la cantidad de glucosa y cloruro de amonio en función de la relación C:N, previamente seleccionada. La FES se evaluó durante 30 días, a $28^{\circ} \mathrm{C}$.

La selección de la mejor cepa fúngica correspondió a aquella con el mayor porcentaje de degradación. Para ello, se realizó, inicialmente, un proceso de desorción, que consistió en la adición de $60 \mathrm{~mL}$ de $\mathrm{KOH} \mathrm{0,005M}$, con agitación de 140rpm, durante 120 minutos y a temperatura ambiente. La cantidad de colorante residual, se cuantificó a partir de la lectura de absorbancia a la longitud de onda de máxima absorción del $A B\left(\lambda_{\text {máx }}=629 \mathrm{~nm}\right)$, determinada en un espectrofotómetro UV/Vis Lambda 35 de doble haz, marca Perkin Elmer, donde el colorante total extraído del control abiótico, sin hongo, se consideró del $100 \%$. A partir de esta información, se calculó el porcentaje de degradación mediante la ecuación 1.

$$
\% \text { Degradación }=\frac{m i-m f}{m f} * 100 \quad \text { Ecuación } 1
$$

Donde:

$m i$ : Masa inicial del colorante adherido, blanco o control (mg) $m f:$ Masa residual de colorante desorbido en la muestra (mg)

En esta investigación, se estableció que una degradación, con un porcentaje cercano al $50 \%$, será considerada poco satisfactoria. En caso de obtenerse dicho valor, se procederá a realizar la FES, utilizando los consorcios de las tres especies fúngicas de estudio.

FES con consorcios: El porcentaje de degradación alcanzado con los consorcios, se determinó a partir de un diseño factorial $2^{3}$, donde los ensayos se llevaron a cabo por triplicado. Los factores considerados fueron factor consorcio, dado por los niveles C1: $P$. ostreatus-P. pulmonarius, C2: $P$. ostreatus-T. versicolor, factor biomasa con niveles de 2 y 4 discos de $1 \mathrm{~cm}$ de diámetro colonizados de hongo y el factor relación C:N, con relaciones de 40:1 y 20:1. Además, se mantuvo la solución de sales del medio Radha, descrita anteriormente en la FES, para cepas individuales. Cabe señalar que la biomasa fue inoculada con discos de cada una de las especies fúngicas, que conformaban el consorcio en relación 1:1. La fermentación, igualmente, fue llevada a cabo durante 30 días, a $28^{\circ} \mathrm{C}$.

Cinética de degradación de AB: Se seleccionó la mejor combinación de factores, que mostraron los mayores porcentajes de degradación de $\mathrm{AB}$, tanto con las cepas individuales como con los consorcios y se repitieron los ensayos, realizando un registro diario de la cantidad del colorante residual, mediante la ecuación 1. 
Actividad enzimática: La actividad de las enzimas fue medida durante la FES de la cinética de degradación, previamente descrita. Para ello, se añadieron $20 \mathrm{~mL}$ de buffer acetato de sodio $50 \mu \mathrm{M}(\mathrm{pH}=5,5)$ y se agitó durante una hora, con el fin de extraer las enzimas del RF en el buffer. El extracto líquido obtenido, se centrifugó y el sobrenadante se utilizó para la determinación de las enzimas. La actividad lacasa, se midió utilizando buffer citrato de sodio $0,1 \mathrm{M}(\mathrm{pH}=3,0)$ y $10 \mathrm{mM}$ del ácido etilbenzothiazoline-6-sulfonico (ABTS), registrando la absorbancia a $\lambda=420 \mathrm{~nm}$, con un coeficiente de extinción $\varepsilon 420=36.000 \mathrm{M}^{-1} \mathrm{~cm}^{-1}$ (Murugesan et al. 2007; Faraco et al. 2009). La actividad manganeso peroxidasa, MnP fue determinada por la oxidación de una solución de $\mathrm{MnSO}_{4} 1,0 \mathrm{mM}$ en buffer malonato de sodio $50 \mathrm{mM}(\mathrm{pH}=4,5)$, en presencia de $\mathrm{H}_{2} \mathrm{O}_{2} 0,1 \mathrm{~m} M$. Se midió la absorbancia a una $\lambda=270 \mathrm{~nm}$, con un coeficiente de extinción molar $\varepsilon 270=11.590 \mathrm{M}^{-1} \mathrm{~cm}^{-1}$ (Murugesan et al. 2007; Faraco et al. 2009). En cuanto a la actividad lignino-peroxidasa, LiP, se utilizó alcohol veratrílico $2 \mathrm{mM}$, buffer tartrato de sodio $50 \mathrm{mM},(\mathrm{pH}=2,5)$ y $\mathrm{H}_{2} \mathrm{O}_{2} 5 \mathrm{mM}$. La absorbancia, se midió a $\lambda=310 \mathrm{~nm}$, con un coeficiente de extinción molar $\varepsilon 310=9.300 \mathrm{M}^{-1} \mathrm{~cm}^{-1}$ (Tychanowycz et al. 2004). La actividad enzimática fue reportada como unidades enzimáticas -U-, por gramo de sustrato seco -gss-, definidas como la cantidad de enzima requerida para producir 1,0 $\mu \mathrm{mol}$ producto/min (Tychanowycz et al. 2004; Murugesan et al. 2007).
Análisis estadístico: Se realizó un análisis de varianza ANDEVA tipo modelo lineal general, con un nivel de confianza del 95\%, a partir de la información obtenida para la degradación en los diseños factoriales, tanto por las cepas individuales como por los consorcios, definiendo, como variable dependiente, el porcentaje de degradación y como variables independientes, los factores hongo, biomasa y relación C:N, utilizando el software estadístico Statgraphics Centurion XV.II versión 16.1.18

\section{RESULTADOS Y DISCUSIÓN}

Pruebas preliminares de degradación de AB: El medio PDA fue descartado para la evaluación de la degradación del $A B$, dado que sólo se evidenció crecimiento de los tres hongos, más no decoloración durante el tiempo de observación (Figura 1A). En cuanto al medio Radha, se observó decoloración y crecimiento para las tres especies seleccionadas: $T$. versicolor, $P$. ostreatus y $P$. pulmonarius, en los diez días de seguimiento (Figura. 1B); por lo anterior, se seleccionó el medio Radha para el posterior proceso de FES. La diferencia en los resultados puede ser atribuida a las características del medio Radha, el cual, por su alto contenido de nutrientes, en particular, hierro, manganeso y zinc, favorece la inducción y la síntesis de las enzimas ligninolíticas de los HPB (Radha et al. 2005).

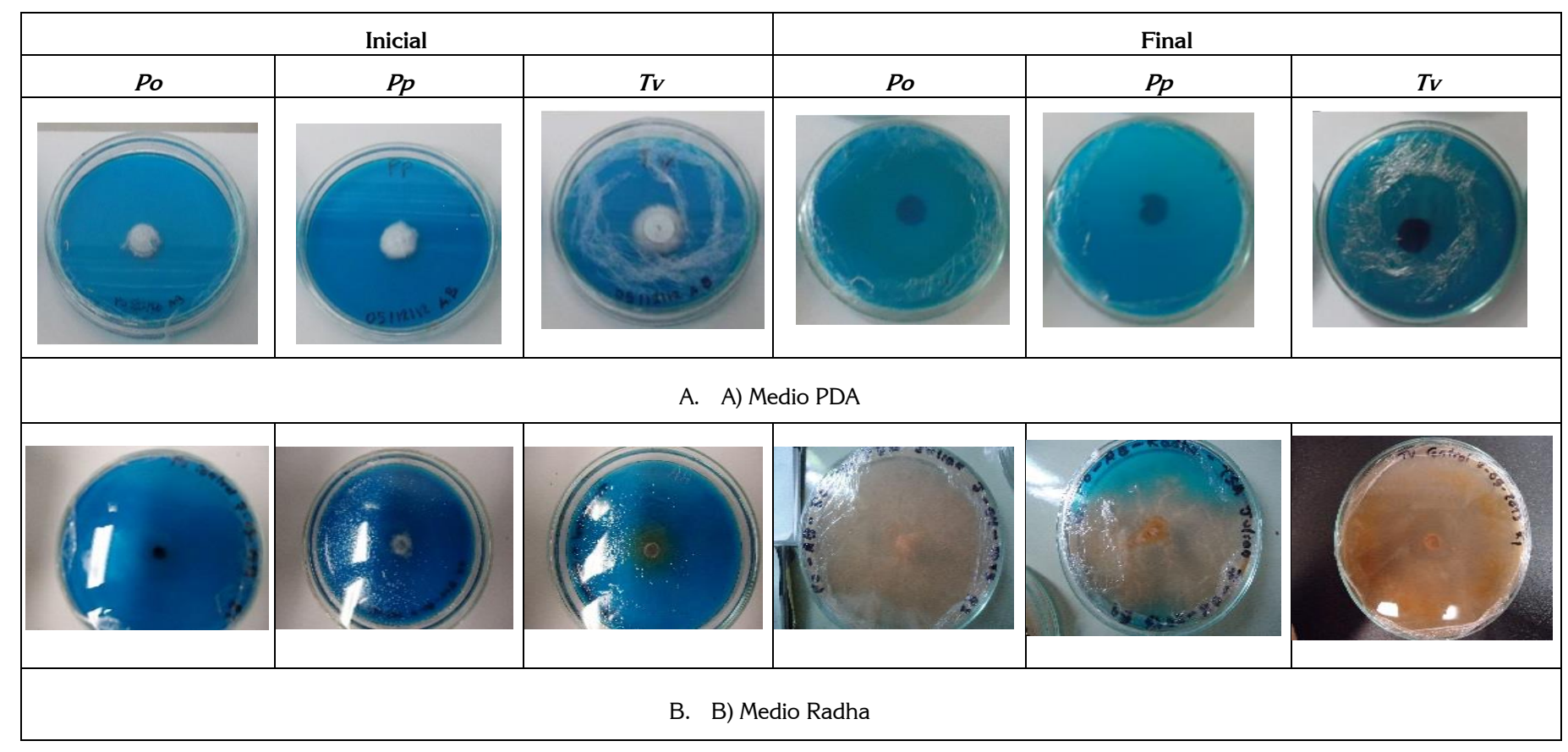

Figura 1. Registro visual de los días inicial y final en la prueba de decoloración del AB, en los dos medios: A) PDA y B) Radha. Pleurotus ostreatus (Po), Pleurotus pulmonarius (Pp), Trametes versicolor (Tv). 
Con respecto a la influencia del cosustrato, se evidenció, visualmente, una desaparición total del color con las tres cepas fúngicas, que fueron suplementadas con glucosa, presentando halos de decoloración, que alcanzaron la totalidad de la caja de Petri, en diez días. En contraste con el ensayo que careció de cosustrato, se observó un crecimiento del hongo con formación de micelio muy delgado, sin percepción visual de la decoloración. Se ha reportado que los HPB mineralizan compuestos complejos, como los colorantes, lo cual, se puede ver favorecido con el aditamento de un cosustrato de carbono de fácil absorción (Radha et al. 2005). Cabe señalar que diferentes carbohidratos han sido probados; sin embargo, la glucosa ha sido la más utilizada, debido a su estructura fácilmente hidrolizable y asimilable (Padmavathy et al. 2003; Koyani et al. 2013).

FES con cepas individuales de HPB: El porcentaje de degradación de $A B$ obtenido con las tres especies evaluadas, se presenta en la tabla 1.

Se observa una diferencia en los porcentajes de degradación entre los dos géneros utilizados, Pleurotus sp. y Trametes sp. De acuerdo al ANDEVA, los hongos utilizados tienen un efecto significativo en la degradación de $A B$, con un valor $F$ de 152,8 , dos grados de libertad y $P<0,05$. De acuerdo a la prueba de Tukey, T. versicolor muestra una diferencia significativa, con un promedio del $53 \%$, con respecto a los dos hongos del género Pleurotus sp., que mantuvieron promedios semejantes y valores de decoloración superiores al $80 \%$.
La baja eficiencia de T. versicolor en la FES puede ser atribuida a la dificultad del hongo para acceder al colorante, que se encuentra adherido al soporte sólido; asimismo y según Boer et al. (2004), se puede deber a la presencia de pigmentos naturales del sustrato, que pueden interferir en los procesos de decoloración o a que el sustrato no suministra las condiciones adecuadas para su crecimiento, tal como una transferencia insuficiente de oxígeno por tamaño de partícula, como lo sugieren Xin \& Geng (2011) y Koyani et al. (2013). Por su parte, P. pulmonarius mostró un alto porcentaje de degradación del 99,14\%, con una relación C:N de 20:1 e indiferente de la biomasa de hongo empleada. Estos resultados concuerdan con los reportados por Tychanowicz et al. (2004), quienes obtuvieron valores superiores al $80 \%$ en la degradación de varios colorantes trifenilmetánicos, utilizando el hongo $P$. pulmonarius, bajo fermentación en estado sólido, con tuza de maíz. De forma general, el contraste en los resultados obtenidos para los dos géneros evaluados puede ser atribuido a las diferencias intrínsecas en su metabolismo (Jaramillo et al. 2014; Rojas \& Hormaza, 2014).

Con respecto al factor $\mathrm{C}: \mathrm{N}$, se alcanzó una degradación satisfactoria, del orden del $86-88 \%$ a cualquier nivel; sin embargo, el proceso de decoloración, se puede mejorar, al combinar el factor biomasa, en un nivel de 4 discos de $1 \mathrm{~cm}$, obteniéndose degradaciones del $96 \%$. Resultados con una eficiencia similar han sido descritos por Keliang et al. (2009), para la decoloración de tres colorantes trifenilmetánicos, mediante FES, sobre cascarilla de arroz.

Tabla 1. Porcentajes de degradación para la FES con las especies fúngicas individuales.

\begin{tabular}{|c|c|c|c|c|}
\hline \multirow[b]{2}{*}{ Hongo } & \multicolumn{2}{|c|}{ Condiciones } & \multirow[b]{2}{*}{ \% Degradación } & \multirow{2}{*}{$\begin{array}{c}\text { Medias } \\
\text { ANDEVA por } \\
\text { Hongo }\end{array}$} \\
\hline & Biomasa (Discos) & Relación C:N & & \\
\hline \multirow{4}{*}{ P. ostreatus } & 2 & 40 & 88,76 & \multirow[b]{4}{*}{94,05} \\
\hline & 4 & 40 & 95,87 & \\
\hline & 2 & 20 & 88,71 & \\
\hline & 4 & 20 & 86,16 & \\
\hline \multirow{4}{*}{ P. pulmonarius } & 2 & 40 & 61,88 & \multirow[b]{4}{*}{86,73} \\
\hline & 4 & 40 & 77,64 & \\
\hline & 2 & 20 & 96,20 & \\
\hline & 4 & 20 & 99,14 & \\
\hline \multirow{4}{*}{ T. versicolor } & 2 & 40 & 57,79 & \multirow[b]{4}{*}{58,21} \\
\hline & 4 & 40 & 60,98 & \\
\hline & 2 & 20 & 42,37 & \\
\hline & 4 & 20 & 51,10 & \\
\hline
\end{tabular}


FES con consorcios de HPB: Los consorcios utilizados en este estudio fueron seleccionados a partir de pruebas de compatibilidad en estudios previos, como los reportados por Rojas \& Hormaza (2014). Los resultados mostrados en la tabla 2, muestran que el C2, P. ostreatus y P. pulmonarius, logró alcanzar una biodegradación del 99,19\%, con una relación de C:N de 40:1 y una biomasa de 4 discos. Esta elevada eficiencia, se explica debido a que las especies fúngicas mostraron una alta capacidad de decoloración del $A B$ de forma individual; asimismo, a que su compatibilidad positiva condujo a una mayor acción metabólica (Chi et al. 2007; Albert et al. 2011).
Para el C1, P. ostreatus y T. versicolor, se obtuvo un valor promedio de degradación del $78 \%$, señalando un aumento del $25 \%$, para T. versicolor, con respecto al resultado obtenido en la FES, de forma individual, del $53 \%$; lo anterior evidencia la favorabilidad del uso de este consorcio en la degradación de colorantes. Resultados similares con consorcios fúngicos han sido registrados por Verma \& Madamwar (2002) y Yang et al. (2011), utilizando un consorcio de Phanerochaete chrysosporium y P. ostreatus y un cultivo mixto de Trametes sp. y Chaetomiun sp., obteniendo porcentajes satisfactorios en la biodegradación de colorantes, los cuales, se encuentran entre el 63 y el $96 \%$.

Tabla 2. Porcentajes de degradación para la FES con los consorcios fúngicos.

\begin{tabular}{|c|c|c|c|c|}
\hline \multirow{2}{*}{ Consorcio } & \multicolumn{2}{|c|}{ Condiciones } & Medias \\
\cline { 2 - 4 } & Biomasa (Discos) & Relación C/N & \% Biodegradación & $\begin{array}{c}\text { ANDEVA por } \\
\text { consorcio }\end{array}$ \\
\hline \multirow{2}{*}{ Consorcio 1 } & 2 & 40 & 88,86 & \\
\cline { 2 - 4 }$($ T. versicolor - & 4 & 40 & 98,76 & \\
\cline { 2 - 4 } P. ostreatus) & 2 & 20 & 55,31 & \multirow{2}{*}{78,28} \\
\hline Consorcio 2 & 4 & 20 & 96,54 & \\
\cline { 2 - 4 }$($ P. ostreatus - & 4 & 40 & 92,57 & \\
\cline { 2 - 4 } & 2 & 40 & 99,20 & \multirow{2}{*}{92,43} \\
\hline
\end{tabular}

El análisis ANDEVA mostró un $P<0,05$ para los factores evaluados, indicando significancia estadística, para cada uno de ellos, al igual que para sus interacciones, resaltando los valores de degradación más altos en una C:N, de 40:1, la cual, difiere de las investigaciones de Pointing et al. (2000) y Herpoël et al. (2000), según, las cuales, los HPB dieron lugar a mayores actividades enzimáticas, bajo condiciones limitantes de nutrientes. En cuanto a la biomasa, la más favorecida, para ambos consorcios, fue la de cuatro discos, permitiendo sugerir que una mayor cantidad de biomasa favorece la producción de enzimas, que participan en la degradación del colorante $\mathrm{AB}$.

Cinética de degradación de AB: Para su análisis, se tomaron las condiciones, bajo las cuales, se obtuvieron los mejores resultados de degradación en la FES. Así, en el caso de las cepas individuales de $P$. ostreatus, una relación C:N de 40:1 y cuatro discos de hongo y para $P$. pulmonarius, una relación $\mathrm{C}: \mathrm{N}$ de 20:1 e, igualmente, con cuatro discos de hongo. Para los consorcios C1 y C2, se tomó la relación C:N 40:1 y una biomasa de cuatro discos de $1 \mathrm{~cm}$.
En la figura 2, se presenta la cinética registrada para las dos especies fúngicas y los dos consorcios, evaluados durante 20 días. Como se observa, el consorcio 2 requirió 10 días para alcanzar una decoloración del 92\% del AB (Figura 2); este mismo porcentaje satisfactorio fue alcanzado a los 12 días, por $P$. pulmonarius y a los 15 días, por el consorcio 1 y por $P$. ostreatus, mostrando claramente una mayor eficiencia por parte del consorcio 2.

De forma general, se aprecia que a medida que transcurre el tiempo de observación aumenta la degradación del colorante, siendo más marcada esta tendencia para el consorcio 2, que pude alcanzar una degradación del 99,0\%, a los 16 días del proceso de FES. Su gran eficiencia, se puede argumentar, debido a la sinergia entre las dos especies fúngicas del mismo género (Albert et al. 2011).

Los resultados obtenidos para la cinética del presente estudio con las cepas individuales superan a los reportados por Tychanowicz et al. (2004) y Robinson et al. (2008), donde se evaluó la degradación de colorantes bajo condiciones simi- 


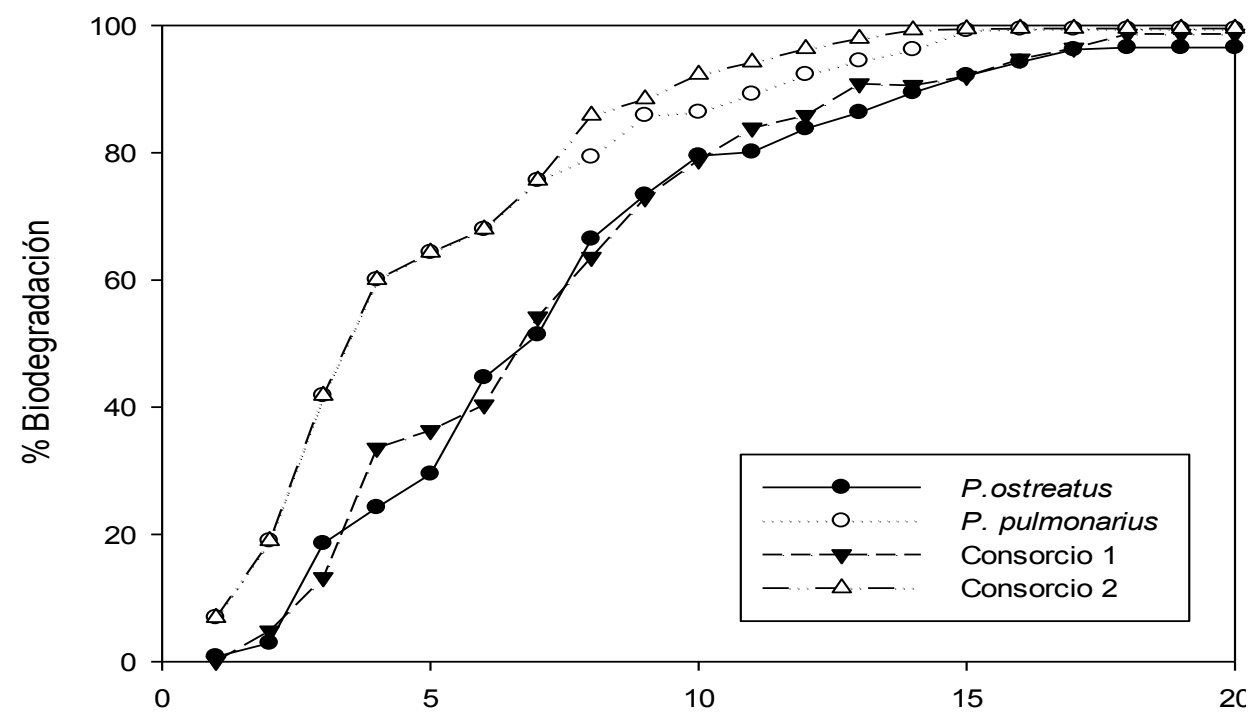

Figura 2. Cinética de degradación de AB, mediante FES.

lares de FES y utilizando los hongos $P$. pulmonarius, en tuza de maíz y Bjerkandera adusta, sobre cáscara de cebada, alcanzando porcentajes de decoloración del 40,0 y 53,1\%, en tiempos de 8 y 21 días, respectivamente. Por otro lado, es de resaltar la alta eficiencia obtenida para los consorcios, con un valor promedio del $92 \%$, a los 12 días.

Actividad enzimática: La medición de la actividad enzimática, se realizó a los 20 días del proceso de fermentación, tiempo necesario para la ocurrencia de la degradación. De acuerdo a la tabla 3, P. pulmonarius, bajo una relación C:N de 20:1 y 4 discos de hongo, registró la presencia de Lacasa, alcanzando un valor de 6,44ULac/gss, mientras que para $P$. ostreatus, bajo una relación de $\mathrm{C} / \mathrm{N}$ de 40:1 y 4 discos de hongo, se determinó un valor de 7,52ULac/gss, es decir, valores prácticamente similares. Al respecto, cabe mencionar, el estudio llevado a cabo por Tychanomicz et al. (2004), bajo condiciones de FES, utilizando $5,0 \mathrm{~g}$, de tuza de maíz y una relación C:N de 30:1, reportando un valor de 30ULac/gss, con el hongo P. pulmonarius. Por su parte, Keliang et al. (2009) obtuvieron, bajo esta metodología, mejores resultados con $P$. ostreatus, utilizando tres colorantes trifenilmetánicos, cristal violeta, azul de bromofenol y verde malaquita, con $5,0 \mathrm{~g}$, de cascarilla de arroz, como soporte sólido. Ellos obtuvieron una máxima producción de Lacasa, igual a 139,6ULac/gss, para el colorante azul de bromofenol.

En cuanto a los consorcios, para la enzima lacasa, se obtuvieron valores de 19,22ULac/gss, para el C1 y 16,45ULac/ gss, para el C2, es decir, no se aprecia una diferencia entre los mismos. Los resultados muestran que la actividad lacasa, registrada en este estudio con los consorcios, supera a los reportados por Carabajal et al. (2012), donde el consorcio formado por los HPB, P. ostreatus y $P$. citrinopileatus, dio lugar a actividades lacasas, con valores de 3,45ULac/gss.

Los elevados porcentajes de degradación obtenidos en la presente investigación para los consorcios y las especies individuales $P$. pulmonarius y $P$. ostreatus, permiten sugerir que la FES representa una metodología biológica alternativa y

Tabla 3. Actividad enzimática en la FES de $\mathrm{AB}$ con especies individuales y consorcios.

\begin{tabular}{|c|c|c|c|}
\hline \multirow{2}{*}{} & \multicolumn{3}{|c|}{ Actividad Enzimática (ULac/gss) } \\
\cline { 2 - 4 } & Lacasa & MnP & LiP \\
\hline Pp & 6,440 & 4,210 & 0,310 \\
\hline Po & 7,525 & 4,081 & 0,291 \\
\hline C1 & 19,229 & 1,395 & 0,319 \\
\hline C2 & 16,450 & 12,191 & 0,294 \\
\hline
\end{tabular}


eficiente para el tratamiento de colorantes trifenilmetánicos, tales como el AB.

El establecimiento de consorcios permitió evidenciar el sinergismo de las especies individuales, mediante un incremento en la producción enzimática que, finalmente, favorece el proceso de degradación del colorante de estudio. Se obtuvieron valores superiores para los consorcios con respecto a las cepas individuales, para las enzimas Lacasa y Manganeso Peroxidasa.

Agradecimientos: Los autores expresan agradecimientos a la Universidad Nacional de Colombia - Sede Medellín- por el apoyo- a través de la infraestructura del Laboratorio de Química Experimental. Financiación: Este estudio fue financiado por la Convocatoria Jóvenes Investigadores e Innovadores 617, año 2013 de Colciencias y por la Universidad Nacional de Colombia - Sede Medellín. Conflicto de intereses: El manuscrito fue preparado y revisado con la participación de todos los autores, quienes declaramos que no existe conflicto de intereses que ponga en riesgo la validez de los resultados presentados.

\section{BIBLIOGRAFÍA}

1. ALBERT, S.; CHAUHAN, D.; PANDYA, B.; PADHIAR, A. 2011. Screening of Trichoderma spp. as potential fungal partner in co-culturing with white rot fungi for efficient bio-pulping. Global J. Biotech. Biochem, (India). 6(3):95-101.

2. BOER, C.G.; OBICI, L.; DE SOUZA, C.G.M.; PERALTA, R.M. 2004. Decolorization of synthetic dyes by solid state cultures of Lentinula (Lentinus) edodes producing manganese peroxidase as the main ligninolytic enzyme. Bioresour. Technol. (Brazil) 94(2):107-112.

3. CARABAJAL, M.; LEVIN, L.; ALBERTO, E.; LECHNER, B. 2012. Effect of co-cultivation of two Pleurotus species on lignocellulolytic enzyme production and mushroom fructification. Int. Biodet. Biodegr. (Argentina) 66(1):71-76.

4. CHANDER, M.; ARORA, D.S. 2007. Evaluation of some white-rot fungi for their potential to decolourise industrial dyes. Dyes Pigments. (India). 72(2):192-198.

5. CHI, Y.; HATAKKA, A.; MAIJALA, P. 2007. Can co-culturing of two white-rot fungi increase lignin degradation and the production of lignin-degrading enzymes? Int. Biodet. Biodegr. 59(1):32-39.

6. ECHAVARRIA, A.M.; HORMAZA, A. 2014. Flower wastes as a low-cost adsorbent for the removal of acid blue 9. DYNA. (Colombia). 81(185):132-138.
7. FARACO, V.; PEZZELLA, C.; MIELE, A.; GIARDINA, P.; SANNIA, G. 2009. Bio-remediation of colored industrial wastewaters by the white-rot fungi Phanerochaete chrysosporium and Pleurotus ostreatus and their enzymes. Biodegradation. (Italia). 20(2):209-220.

8. FERNÁNDEZ, J.A.; HENAO, L.M.; PEDROZA RODRÍGUEZ, A.M.; QUEVEDO HIDALGO, B. 2009. Inmovilización de hongos ligninolíticos para la remoción del colorante negro reactivo 5. Rev. Col. Biotecn. 11(1):59-72.

9. GUPTA, V.K.; MITTAL, A.; KRISHNAN, L.; MITTAL, J. 2006. Adsorption treatment and recovery of the hazardous dye. Brilliant Blue FCF over bottom ash and de-oiled soya. J. Colloid Interface Sci. (India) 293(1): 16-26.

10. GUTIÉRREZ-CORREA, M.; PORTAL, L.; MORENO, P.; TENGERDY, R.P. 1999. Mixed culture solid substrate fermentation of Trichoderma reesei with Aspergillus niger on sugar cane bagasse. Bioresour. Technol. (Perú-Estados Unidos). 68:173-178.

11. GUTIÉRREZ, M.F.; BONILLA, M. DEL P.; ESPINOSA, A.; MOSQUERA, M.; SOLANO, S.; MARTÍNEZ, M.M. 2009. Presencia de rotavirus durante un proceso de compostaje. Abonos como vectores de contaminación viral. Univers. Scient. (Colombia) 14(2-3):206215.

12. HERPOËL, I.; MOUKHA, S.; LESAGE-MEESSEN, L.; SIGOILLOT, J.; ASTHER, M. 2000. Selection of Pycnoporus cinnabarinus strains for laccase production. FEMS Microbiol. Lett. (Francia). 183(2):301-306.

13. JARAMILLO, A.; JIMÉNEZ, S.; MERINO, A.; HORMAZA, A. 2014. Obtención de un inóculo fúngico para la degradación de un colorante azo por fermentación en estado sólido. Rev. U.D.C.A Act. \& Div. Cient. (Colombia). 17(2):577-585.

14. JARAMILLO, A.C.; ECHAVARRÍA, A.M.; HORMAZA, A. 2013. Diseño Box-Behnken para la optimización de la adsorción del colorante azul ácido sobre residuos de flores. Rev. Ing. Ciencia. (Colombia). 9(18):75-91.

15. KAUSAR, H.; SARIAH, M.; MOHD SAUD, H.; ZAHANGIR ALAM, M.; RAZI ISMAIL, M. 2010. Development of compatible lignocellulolytic fungal consortium for rapid composting of rice straw. Int. Biodet. Biodegr. (Malasia). 64(7):594-600. 
16. KAUSHIK, P.; MALIK, A. 2009. Fungal dye decolourization: Recent advances and future potential. Environt. Int. (India). 35(1):127-141.

17. KELIANG, Y.; WANG, H.; ZHANG, X.; YU, H. 2009. Bioprocess of triphenylmethane dyes decolorization by Pleurotus ostreatus BP under solid-state cultivation. J. Microbiol. Biotechnol. (China). 19(11):1421-1430.

18. KOYANI, R.D.; SANGHVI, G.V.; SHARMA, R.K.; RAJPUT, K.S. 2013. Contribution of lignin degrading enzymes in decolourisation and degradation of reactive textile dyes. Int. Biodet. Biodegr. (India) 77:1-9.

19. MALAVIYA, P.; RATHORE, V.S. 2007. Bioremediation of pulp and paper mill effluent by a novel fungal consortium isolated from polluted soil. Biores. Techn. (India). 98(18):3647-3651.

20. MURUGESAN, K.; NAM, I.H.; KIM, Y.M.; CHANG, Y.S. 2007. Decolorization of reactive dyes by a thermostable laccase produced by Ganoderma lucidum in solid state culture. Enzyme Microb. Technol. (India). 40(7):1662-1672.

21. PADMAVATHY, S.; SANDHYA, S.; SWAMINATHAN, K.; SUBRAHMANYAM, Y.; CHAKRABARTI, T; KAUL, S.N. 2003. Aerobic decolorization of reactive azo dyes in presence of various cosubstrates. Chem. Biochem. Eng. Q. (India). 7(2):147-151.

22. POINTING, S.B.; ROAD, P.; AVENUE, T.C. 2000. Decolorization of azo and triphenylmethane dyes by Pycnoporus sanguineus producing laccase as the sole phenoloxidase. World J. Microbiol. Biotechn. (China). 16:317-318.

23. PRIGIONE, V.; VARESE, G.C.; CASIERI, L.; MARCHISIO, V.F. 2008. Biosorption of simulated dyed effluents by inactivated fungal biomasses. Biores. Techn. (Italia). 99(9):3559-3567.

24. RADHA, K.V.; REGUPATHI, I.; ARUINAGIRI, A.; MURUGESAN, T. 2005. Decolorization studies of synthetic dyes using Phanerochaete chrysosporium and their kinetics. Process Biochem. (India). 40(10):3337-3345.

25. ROBINSON, T.; NIGAM, P.S. 2008. Remediation of textile dye waste water using a white-rot fungus Bjerkan- dera adusta through solid-state fermentation (SSF). Appl. Biochem. Biotechnol. (Reino Unido) 151(23):618-628.

26. ROBINSON, T.; MCMULLAN, G.; MARCHANT, R.; NIGAM, P. 2001. Remediation of dyes in textile effluent: a critical review on current treatment technologies with a proposed alternative. Bioresour. Technol. (Reino Unido). 77:247-255.

27. ROJAS, J.A.; HORMAZA, A. 2014. Evaluación del crecimiento y compatibilidad de hongos de la podredumbre blanca. Ciencia Desarrollo. (Colombia). 5(2):197-205.

28. SARATALE, R.G.; SARATALE, G.D.; KALYANI, D.C.; CHANG, J.S.; GOVINDWAR, S.P. 2009. Enhanced decolorization and biodegradation of textile azo dye Scarlet R by using developed microbial consortiumGR. Biores. Techn. (India-Taiwan) 100(9):2493-2500.

29. TYCHANOWICZ, G.; ZILLY, A.; DE SOUZA, C.G.M.; PERALTA, R. 2004. Decolourisation of industrial dyes by solid-state cultures of Pleurotus pulmonarius. Process Biochem. (Brazil). 39:855-859.

30. VERMA, P.; MADAMWAR, D. 2002. Production of ligninolytic enzymes for dye decolorization by cocultivation of white-rot fungi Pleurotus ostreatus and Phanerochaete chrysosporium under solid-state fermentation. Appl. Biochem. Biotechnol. (India). 102-103(16):109-118.

31. XIN, F.; GENG, A. 2011. Utilization of horticultural waste for laccase production by Trametes versicolor under solid-state fermentation. Appl. Biochem. Biotechnol. (Singapur). 163(2):235-246.

32. YANG, X.; WANG, J.; ZHAO, X.; WANG, Q.; XUE, R. 2011. Increasing manganese peroxidase production and biodecolorization of triphenylmethane dyes by novel fungal consortium. Biores. Techn. (China). 102(22):10535-10541.

Recibido: Agosto 2 de 2015

Aceptado: Diciembre 26 de 2015

Cómo citar:

Rojas, J.; Hormaza A. 2016. Evaluación de la biodegradación del colorante azul brillante utilizando hongos de la podredumbre blanca y sus consorcios. Rev. U.D.C.A. Act. \& Div. Cient. 19(1): 179-187. 